\title{
Role Of Anaesthetist In Sedation For Diagnostic Procedures: The Glass Is Half Empty? A Narrative Review
}

\author{
Alberto Pasqualucci ${ }^{1 *}$,Mansour Yousef Nadhari ${ }^{2}$, Kurian Palavilayil Thomas Vaidyan $^{2}$, Giustino Varrassi ${ }^{3}$, \\ Jo Ann LeQuang ${ }^{4}$, Joseph V Pergolizzi ${ }^{4}$ and Antonella Paladini ${ }^{5}$ \\ ${ }^{1}$ Department of Scienze Chirurgiche e Biomediche, Università degli Studi di Perugia, Italy \\ ${ }^{2}$ Department of Anesthesia and Emergency, Rashid Hospital (Dubai Health Authority), Dubai, UAE \\ ${ }^{3}$ Paolo Procacci Foundation, Roma, Italy \\ ${ }^{4}$ NEMA Research Group, Naples, FL, USA \\ ${ }^{5}$ Department of MESVA, University of L'Aquila, L'Aquila, Italy
}

Received: October 06, 2017; Accepted: October 31, 2017, Published: November 10, 2017

*Corresponding author: Alberto Pasqualucci, Rashid Hospital, Trauma and Emergency Center (DHA), 315 HMM HURAIR Second, PO Box 4545 Dubai, UAE, Tel.: 0755783429 and 075 5783594; E-mail: alberto.pasqualucci@unipg.it

\begin{abstract}
Sedation of outpatients undergoing diagnostic and therapeutic procedures remains a controversial topic, and there remains a paucity of literature and guidelines to help inform sound clinical decisions. As these are frequently performed procedures that offer advantages to patients in terms of their comfort and well-being, we aimed to better address this information deficit and to clarify why appropriate guidelines are still not available. To this end, we propose reviewing sedation procedures performed using Magnetic Resonance Imaging (MRI) in pediatric patients.
\end{abstract}

Keywords: Sedation; Diagnostic Procedure; Paediatric Patients; Magnetic Resonance Imaging (MRI); Guidelines for Sedation; Evaluation Scales;

\section{Introduction}

Sedation of outpatients undergoing diagnostic and therapeutic procedures remains controversial. Despite the increase in the last few years in the number and complexity of such outpatient procedures, there is limited scientific data and no guidelines to help describe safe, optimal sedation. The few scientific publications on procedural sedation address such things as standardization of processes and reasons for sedation [1-5]. Given the frequency and importance of these procedures in terms of the comfort and well-being of patients, we have decided to review the literature with the goal of establishing the reason(s) that "appropriate" guidelines have thus far not been issued.

Our analysis has concentrated on sedation procedures performed to facilitate diagnostic procedures like Magnetic Resonance Imaging (MRI), Computerized Tomography (CT scans) etc., in pediatric patients. Following this analysis we have tried, briefly, to suggest some solutions that could aid the formulation of universal guidelines. It is our goal to establish a research protocol based on clinical experiences with these pediatric patients.

\section{Methods}

This is a narrative review. The authors examined the literature related to sedation procedures, standard processes, and reasons for sedation. There are no formal guidelines issued for sedation. It is the long-range goal of the authors to establish a research protocol to gain the necessary clinical experience to further elucidate this important subject. To this end, the narrative review begins the exploration of this topic and lays the groundwork for future clinical study. The proposed research protocol will presumably use pediatric patients from two centers (likely Rashid Hospital in Dubai and the University of L'Aquila in Italy) in the approximate age range of 0 to 8 years. Pediatric patients have been selected because they usually need sedation to undergo magnetic resonance imaging Problems

Outpatient anesthesia faces two separate but interconnected main issues. They can be defined as "scientific-clinical" and "technical-organizational."

The scientific-clinical aspect entails the following:

1.Much of the literature on the subject of outpatient sedation reports on retrospective non-randomized trials or papers about the pharmacology of sedative agents [6-9].

2.The literature reveals differences among hospitals and sometimes within one hospital as to the way to deliver sedation. The choice of technique and sedative drugs is usually left to the operator [5,7,10-12].There is little agreement in terms of the techniques (spontaneous breathing versus general anesthesia; laryngeal mask versus endotracheal tube), choice of drugs, and how those drugs are to be administered (intravenous, rectal, etc.) $[5,7,10-12]$. 
3.There is no agreement on the utilization and validity of various measurement scales that evaluate the phases of sedation [13].

4.In some papers, measurement scales are not mentioned at all $[5,8,13,14]$.

The "technical-organizational" issues have emerged in the last few years due to a geometrical increase of the number of requests for sedation and their associated costs. An increased number of procedures has produced an increased demand for anesthetists that has resulted in substantially increased costs for these procedures. In order to meet demand but still contain costs, a compromise solution has taken root. This compromise consists of allowing medical personnel not trained in anesthesiology to administer sedative drugs $[5,7,11,14]$. Furthermore, in recent years, some countries have given permission to nurses, paramedics, and other healthcare professionals to administer sedatives. In our opinion, this has increased the number of scientific-clinical issues.

As a result, the attention of the researchers on the topic of sedation has been diverted from the procedure itself. Some studies have been designed to establish who, among the healthcare personnel, can appropriately deliver sedative drugs, which drugs to use and their route of administration in various procedures. This shift between the above-mentioned issues has favoured the technical-organizational rather than the scientificclinical issue $[5,15,16]$. This choice by researchers has brought more confusion and variability to the available published data and makes them difficult to compare. Indeed, these data have been produced by different healthcare professionals utilizing different techniques. Some of them have different skills in the management of the airways, depth of sedation, hemodynamic derangement caused by drugs, and overall protection of the sedated patient [5,11,12,15-18].

During a sedation procedure (where the main objective is to achieve the patient's immobility and, in some cases, deep analgesia) the level of confidence/experience of the operator in the utilization of sedative and analgesic drugs, together with knowledge of drug actions is crucial to patient safety; this should be the basic standard used in the future to compare techniques, drugs and modes of administration in sedative procedures $[5,11,12,15-18]$.

Some studies (and indeed routine clinical practice) do not always specify minimum required skills for the healthcare professional delivering sedation, and in some cases, even anesthesia may be administered by someone other than a trained and skilled anesthetist. As a result, it could be argued that some drugs and techniques have not been optimally employed [5,11,12,14-18].This argument is further supported by the fact that in multiple papers, complications associated with sedative procedures have been described, which necessitated help from an anesthetist [5,11,12,14-18].

Even when well-trained healthcare professionals are used, their lack of specific training and experience in sedation may well have affected the results of various studies $[5,11,12,14-16,18]$.

\section{Proposal for Solution}

Robust guidelines must be based on sound medical evidence, which in this case can only be obtained by standardizing how sedation is delivered. The optimal delivery of sedation is accomplished by clinician strained in anesthesia. Their experience would allow them to identify and utilize the best available drugs and techniques to full potential to obtain the optimal outcome $[6-8,10,12,15,19,20]$. This would enhance patient safety and also result in studies yielding data with comparable methodologies, skills, and techniques.

Concerning the problems about the sedation evaluation scales, we support the indications of Williams and collaborators13.The authors, after careful comparison and analysis of various sedation measurement scales, point out that, until now, there has been a lack consistency among various validation tools used during before, during, and after procedures. The Ramsay score 21 has been recommended; itis a well-known and frequently applied scoring system, used for documentation of adequate depth of sedation $[7,13,20,22]$.

In the domain of clinical and sedation efficacy/quality, the satisfaction of the physician performing the procedure must be assessed in addition to the clinician who deliversthe sedation. The literature seems to indicate that, for example, the result of sedation for anMRI scan could be evaluated/graded for quality on a 3-point scale: (i) excellent quality, that is, no motion artifact; (ii) minor motion, scan completed; or (iii) major motion; scan incomplete [18].

For documentation of adequate recovery after the procedure, it the wide spread Modified Aldrete score 23 used for determination of discharge criteria could be applied ${ }^{12-13,22}$. Detection of an emergence delirium could also be performed with the Pediatric Anesthesia Emergence Delirium (PAED) scale during recovery time for a better understanding prolonged emergence [17,24,25].

\section{Discussion}

Two parallel conditions describe the situation in which outpatient sedation is currently administered: more and more, non-anesthesiologists are administering outpatient sedation and there remains a paucity of literature and no guidelines to advise them as to the appropriate protocols. These two trends are inter-related. The use of non-anesthesiologists to administer and manage outpatient sedation often means that drugs and techniques may vary widely among institutions (and even within an institution) and may not always represent the optimized use of the available agents and methods. As a result, studies of outpatient sedation yield data that are difficult to compare because different facilities use different drugs, different routes of administration, and so on, for the same procedures. Without comparable data on which to draw conclusions, arriving at scientifically sound guidelines is nearly impossible. Our study, which was based on studies limited to the field of pediatric diagnostic imaging procedures, shows what we think is a trend across all fields of outpatient procedures. Non-anesthesiologists often administer 
outpatient sedation and currently drugs, techniques, and routes of administration vary so widely that robust data for guidelines simply are not available.

Although it may seem difficult to remedy, it is easy to understand how this situation arose. Outpatient procedures have increased markedly in recent years; they are generally more economical than inpatient procedures and patients tend to prefer the convenience of day surgery. Outpatient procedures make financial sense for providers, clinics, payers, and patients. Patients like them and they facilitate the all-important daily logistics of a busy clinic. With this sudden surge in in-patient procedures came an increased demand for those who could administer sedation in the outpatient setting. It seems more cost efficient to utilize the services of a non-anesthesiologist, and there is even some support for this approach. Certain healthcare professionals could reasonably be trained to administer sedation in the outpatient setting and the use of these non-anesthesiologist as been effective in containing costs. The problem arises that these healthcare professionals would likely not be as well informed about sedative agents, analgesics, and sedation techniques as an anesthesiologist; in the event of complications or a difficult case, these healthcare professionals might lack the appropriate skills to manage a crisis. This problem might be at least somewhat ameliorated by the existence of clear evidence-based guidelines on outpatient sedation. However, our current state of haphazard protocols (different healthcare professionals administer outpatient sedation in different ways at different institutions) has hampered our ability to capture reasonable and reliable data that can aid in the formulation of such guidelines.

We advocate that outpatient procedures rely on the services of anesthesiologists and these specialists work together to use the same validated measurement tools to quantify sedation levels and determine discharge criteria. These specialists should publish their work to contribute to a body of literature that can be used to better define protocols and set up guidelines for outpatient sedation. The experience and expertise of anesthesiologists is urgently needed so that these guidelines can be defined. This is not a clinical nicety, it is necessary to guard patient safety.

\section{Conclusion}

The recent rise in outpatient procedures has resulted in two trends: non-anesthesiologists frequently administer outpatient sedation in these settings and there is limited literature and no guidelines to help determine these protocols. In our limited study of outpatient sedation involving pediatric patients undergoing imaging procedures, we defined clinical and technical obstacles to outpatient anesthesia and we advocate for the use of anesthesiologists to administer outpatient sedation as well as to use validated measurement tools and publish their findings. This would provide the foundation for the publication of scientifically sound evidence-based guidelines on outpatient sedation.

\section{References}

1. American Society of Anesthesiologists. Practice guidelines for sedation and analgesia by non-anesthesiologists. Anesthesiology 2002;96(4):1004-1017.

2. Leffler DA, Bukoye B, Sawhney M, Berzin T, Sands K, Chowdary S, et al. Development and validation of the PROcedural Sedation Assessment Survey (PROSAS) for assessment of procedural sedation quality. GastrointestEndosc. 2015; 81(1):194-203.e1. doi: 10.1016/j.gie.2014.07.062

3. Coté CJ. American Academy of Pediatrics sedation guidelines: are we there yet? Arch PediatrAdolesc Med. 2012;166(11):1067-1069. doi: 10.1001/archpediatrics.2012.1355

4. Godwin SA, Burton JH, Gerardo CJ, Hatten BW, Mace SE, Silvers $\mathrm{SM}$, et al. Clinical policy: procedural sedation and analgesia in the emergency department. Ann Emerg Med. 2014;63(2):247-258.e18. doi: 10.1016/j.annemergmed.2013.10.015

5. Kamat, PP, Hollman GA, Simon HK, Fortenberry JD, McCracken CE, Stockwell JA. Current State of Institutional Privileging Profiles for Pediatric Procedural Sedation Providers. Hosp Pediatr. 2015;5(9):487-494. doi: 10.1542/hpeds.2015-0052

6. Fang H, Yang L, Wang X, Zhu H. Clinical efficacy of dexmedetomidine versus propofol in children undergoing magnetic resonance imaging: a meta-analysis. Int J ClinExp Med 2015;8(8):11881-11889.

7. Schulte-Uentrop L, Goepfert MS. Anaesthesia or sedation for MRI in children. Curr Opin Anaesthesiol. 2010;23(4):513-517. doi: 10.1097/AC0.0b013e32833bb524

8. Cravero JP, Blike GT. Pediatric sedation. Curr Opin Anaesthesiol. 2004;17(3):247-251.

9. Hansen TG. Sedative medications outside the operating room and the pharmacology of sedatives. Curr Opin Anesthesiol. 2015;28(4):446452. doi: 10.1097/AC0.0000000000000202

10. Usher A, Kearney R. Anesthesia for magnetic resonance imaging in children: a survey of Canadian pediatric centres. Can J of Anesthesia. 2003;50(4):425. DOI: 10.1007/BF03021048

11. Cravero JP, Beach ML, Blike GT, Gallagher SM, Hertzog JH. The incidence and nature of adverse events during pediatric sedation/anesthesia with propofol for procedures outside the operating room: a report from the pediatric sedation research consortium. Anesth Anlalg. 2009;108(3):795-804. doi: 10.1213/ ane.0b013e31818fc334.

12. Usher AG, Kearney RA, Tsui BC. Propofol total intravenous anesthesia for MRI in children. Pediatr Anaesth 2005;15(1):23-28. doi: 10.1111/j.1460-9592.2004.01390.x

13. Williams MR, McKeown A, Dexter F, Miner JR, Sessler DI, Vargo J, et al. Efficacy outcome measures for procedural sedation clinical trials in adults: an acttion systematic review. Anesth Analg. 2016;122(1):152-170. doi: 10.1213/ANE.0000000000000934

14. Malviya S, Voepel-Lewis T, Ludomirsky A, Marshall J, Tait AR. Can we improve the assessment of discharge readiness? A comparative study of observational and objective measures of depth of sedation in children. Anesthesiology. 2004;100(2):218-224. 
15. Mason KP, Sanborn P, Zurakowski D, Karian VE, Connor L, Fontaine PJ, et al. Superiority of pentobarbital versus chloral hydrate for sedation in infants during imaging. Radiology. 2004;230(2):537542. doi: 10.1148/radiol.2302030107

16. Srinivasan M, Turmelle M, DePalma LM, Mao J, Carlson DW. Procedural Sedation for Diagnostic Imaging in Children by Pediatric Hospitalists using Propofol: Analysis of the Nature, Frequency, and Predictors of Adverse Events and Interventions. J Pediatr. 2012; 160(5):801-806.e1. doi: 10.1016/j.jpeds.2011.11.003

17. Cauld well C. Anesthesia risks associated with pediatric imaging. Pediatr Radiol. 2011;41(8):949-950.

18. Malviya S, Voepel-Lewis T, Tait AR, Reynolds PI, Gujar SK, Gebarski SS, et al. Pentobarbital vs chloral hydrate for sedation of children undergoing MRI: efficacy and recovery characteristics. Pediatr Anaesth. 2004;14(7):589-595. doi: 10.1111/j.14609592.2004.01243.x

19. Dalal PG, Murray D, Cox T, McAllister J, Snider R. Sedation and anesthesia protocols used for magnetic resonance imaging studies in infants: provider and pharmacologic considerations. Anesth Analg. 2006;103(4):863-868. doi: 10.1213/01.ane.0000237311.15294.0e
20. Pershad J, Wan J, Anghelescu DL. Comparison of propofol with pentobarbital/midazolam/fentanyl sedation for magnetic resonance imaging of the brain in children. Pediatrics. 2007;120(3):e629-636. doi: 10.1542/peds.2006-3108

21. Ramsay MA, Savege TM, Simpson BR, Goodwin R. Controlled sedation with alphaxalone-alphadolone. Br Med J. 1974;2(5920):656-659.

22. Godoy ML, Pino AP, Córdova LG, Carrasco OJA, Castillo MA. Sedation and analgesia in children undergoing invasive procedures. Arch Argent Pediatr. 2013;111(1):22-8. doi: 10.5546/aap.2013.22

23. Aldrete J. The post-anesthesia recovery score revisited. J Clin Anaesth. 1995;7(1):89-91.

24. Sikich N, Lerman J. Development and psychometric evaluation of the pediatric anesthesia emergence delirium scale. Anesthesiology. 2004;100(5):1138-1145

25. Pieters BJ, Penn E, Nicklaus P, Bruegger D, Mehta B, Weatherly R. Emergence delirium and postoperative pain in children undergoing adenotonsillectomy: a comparison of propofol vs sevoflurane anesthesia. Paediatr Anaesth. 2010;20(10):944-950. doi: 10.1111/j.1460-9592.2010.03394.x 